\section{Safety and efficacy of ibrutinib in a patient with severe renal impairment}

\section{Kalman Filanovsky, Lev Shvidel \\ Kaplan Medical Center, Rehovot; Hadassah and Hebrew University Medical School, Jerusalem, Israel}

Ibrutinib (Imbruvica) is a first-in-class oral inhibitor of Bruton's tyrosine kinase (BTK), an essential enzyme in B-cell receptor signaling pathway. Since recent approval by the U.S. Food and Drug Administration and European Medicines Agency, it has been widely applied in the therapy of patients with lymphoproliferative disorders, such as mantle cell lymphoma, chronic lymphocytic leukemia, and Waldenström's macroglobulinemia. ${ }^{1}$ Many of these patients are elderly and have comorbidities including impaired renal function. ${ }^{2,3}$ There are scarce data in the literature regarding safety and efficacy of the drug in this cohort of patients. ${ }^{4}$ Despite the fact that less than $10 \%$ of ibrutinib is excreted by kidneys, increase in creatinine level 1.5 to 3 times the upper limit of normal occurs in up to $9 \%$ of patients during treatment ${ }^{4}$ and occasionally patients were developed serious renal failure. ${ }^{5}$ Moreover, patients with preexisting renal impairment were generally excluded from clinical trials using this drug. ${ }^{4,6}$ Herein, we describe a 78year-old male with severe chronic renal failure (creatinine level $4.46 \mathrm{mg} / \mathrm{dL}$ (normal, $0.5-0.9) \mathrm{CrCl} 13 \mathrm{~mL} / \mathrm{min}$ and mantle cell lymphoma that relapsed five years after the first complete remission induced by treatment with cyclophosphamide, oncovin, hydroxydoxorubicin, rituximab and prednisone (CHOP-R regimen). Additional biochemical tests such as electrolytes, uric acid and lactate dehydrogenase were within a normal limit. The patient had a transitional cell carcinoma of the ureter four years ago that was successfully treated by partial right ureterectomy followed by bacillus Calmette-Guerin (BCG) vaccine therapy, although creatinine level gradually increased to $2.0 \mathrm{mg} / \mathrm{dL}$. Abdominal ultrasonography demonstrated normal kidney size and structure. Pretreatment positron-emission computer tomography (CT-PET) revealed widespread lymphadenopathy with high ${ }^{18}$ fluorodeoxyglucose (FDG) uptake in nasopharynx, mediastinal lymph nodes and duodenum. Bone marrow was not involved. Treatment was started with standard ibrutinib dose $560 \mathrm{mg}$ once daily and tumor lysis syndrome prophylaxis by intensive oral hydration and allopurinol. Weekly renal function assessment was performed. During the first three mounts of treatment any adverse event was observed; repeated CTPET showed improvement with no FDG avid disease. Moreover, creatinine level after initiation of the treatment rapidly decreased (Figure 1). This finding suggests that additional renal function deterioration caused by disease progression.

In conclusion, our case showed that ibrutinib can be safely used in patients with severe renal failure under close renal function monitoring. Additional clinical post marketing evaluation needs to be performing in order to confirm our observation.
Rapidly improvement renal function after initiation of Ibrutinib

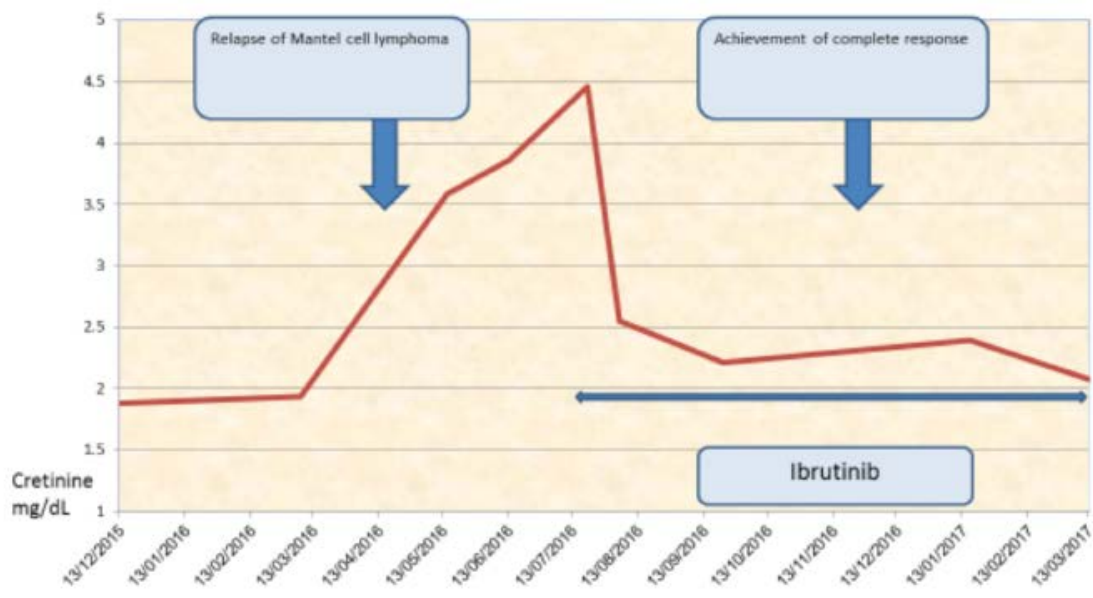

Correspondence: Kalman Filanovsky, Department of Hematology, Kaplan Medical Center, Rehovot; Hadassah and Hebrew University Medical School, P.O.B. 12272, Jerusalem, Israel.

Tel: 97289441383 , Fax: 97289441706

Email:kalmanph@clalit.org.il.

Key words: lymphoma, ibrutinib, chronic renal failure.

Contributions: the authors contributed equally.

Conflict of interest: the authors declare no potential conflict of interest.

Received for publication: 4 February 2017. Revision received: 25 March 2017.

Accepted for publication: 18 April 2017.

This work is licensed under a Creative Commons Attribution-NonCommercial 4.0 International License (CC BY-NC 4.0).

(C) Copyright K. Filanovsky and L. Shvidel, 2017 Licensee PAGEPress, Italy

Hematology Reports 2017; 9:7078

doi:10.4081/hr.2017.7078

\section{References}

1. Castillo JJ, Treon SP, Davids MS. Inhibition of the bruton tyrosine kinase pathway in b-cell lymphoproliferative disorders. Cancer J 2016;22:34-9.

2. Jemal A, Siegel R, Xu J, Ward E. Cancer statistics, 2010. CA Cancer J Clin 2010;60:277-300.

3. Stevens L, Viswanathan G, Weiner D. Chronic kidney disease and end-stage renal disease in the elderly population: current prevalence, future projections, and clinical significance. Adv Chronic Kidney Dis 2010;17:293-301.

4. Wang M, Blum K, Martin P, et al. Longterm follow-up of MCL patients treated with single-agent ibrutinib: updated safety and efficacy results. Blood 2015;126:739-45.

5. Imbruvica ${ }^{\circledR}$ (ibrutinib) capsules [prescribing information]. Sunnyvale, CA: Pharmacyclics; revised June 2016. Available from: https://www.janssen$\mathrm{md}$. c o m/pd f/ i mbruvi ca/P I Imbruvica.pdf

6. Dreyling M, Jurczak W, Jerkeman M, et al. Ibrutinib versus temsirolimus in patients with relapsed or refractory mantlecell lymphoma: an international, randomised, open-label, phase 3 study. Lancet 2016 20;387:770-8.

Figure 1. Rapidly improvement of renal function after the initiation of ibrutinib. 